\title{
A CHARACTERIZATION OF THE CONTACT LIE ALGEBRAS
}

\author{
THOMAS B. GREGORY
}

\begin{abstract}
We classify the simple finite-dimensional irreducible graded Lie algebras over an algebraically closed field of characteristic $p>5$ which have the form $L_{-2} \oplus L_{-1} \oplus L_{0} \oplus L_{1} \oplus \cdots \oplus L_{k}, k>3$, where $L_{0}$ is classical and reductive. We show that any such Lie algebra must be a Lie algebra of the contact series of Lie algebras of Cartan type by showing how the constraints imposed by the hypotheses force the existence of a highest-weight vector in $L_{-1}$ for the representation of $L_{0}$ in $L_{-1}$ induced by the adjoint representation of $L$ in itself. The existence of this highest-weight vector enables us to conclude that the above-mentioned representation is restricted. $L$ can then be determined by appeal to an earlier classification theorem.
\end{abstract}

In [1], the present author classified the simple finite-dimensional graded Lie algebras over an algebraically closed field of characteristic $p>3$ which have the form $L_{-1} \oplus L_{0} \oplus L_{1} \oplus \cdots \oplus L_{k}$ where $L_{0}$ is classical and reductive. In this paper we classify the simple finite-dimensional irreducible graded Lie algebras over an algebraically closed field of characteristic $p>5$ which have the form

$$
L_{-2} \oplus L_{-1} \oplus L_{0} \oplus L_{1} \oplus \cdots \oplus L_{k}, \quad k>3 \text {; }
$$

here, too, we assume that $L_{0}$ is classical and reductive. We in fact prove the following

THEOREM. Let $L$ be a simple finite-dimensional irreducible graded Lie algebra of the form (1) over an algebraically closed field of characteristic $p>5$, and suppose that $L_{0}$ is classical and reductive; that is, that $L_{0}$ is the direct sum of simple, classical Lie subalgebras and a center of dimension at most one. Then $L$ is a Lie algebra of the contact series of Lie algebras of Cartan type.

REMARK. Let $\rho$ be the representation of $L_{0}$ in $L_{-1}$ which is induced by the adjoint representation of $L$ in itself. When we say that $L$ is irreducible, we mean that $\rho$ is irreducible; that is, $L_{-1}$ is a nonzero $L_{0}$-module which contains no proper $L_{0}$-submodules. (When $L_{-1}=\{0\}$, it is easy to see-e.g., from the proof of Lemma 1 below-that $L_{i}=\{0\}$ for all odd $i$, so that $L$ has the form of the Lie algebras classified in [1].) When $\rho$ is a $p$-representation, the above theorem is a consequence of Theorem 3 of [2]. This paper is devoted to showing, by a series of lemmas, that the assumption of restrictedness need not be made in this case.

We fix a system of simple (positive) roots $\left\{\alpha_{i}\right\}_{i=1}^{n}$ of $L_{0}$, and we denote by $B^{+}$ and $B^{-}$the Borel subalgebras associated with the sets of positive and negative

Received by the editors July 22, 1980 and, in revised form, September 17, 1980.

AMS (MOS) subject classifications (1970). Primary 17B20. 
roots, respectively, which are determined by this choice of simple roots. We select root vectors $e_{i}, f_{i}$, and $h_{i}$, associated with the roots $\alpha_{i},-\alpha_{i}$, and 0 , respectively, such that $\left[e_{i}, f_{j}\right]=\delta_{i j} h_{i},\left[e_{i}, h_{i}\right]=2 e_{i}$, and $\left[f_{i}, h_{i}\right]=-2 f_{i}, 1<i<n$.

LEMMA 1. If $L$ is a simple, graded Lie algebra of the form (1), then $L_{-2}$ is an irreducible $L_{0}$-module. Furthermore, $\left[L_{-1}, L_{-1}\right] \neq\{0\}$, so $\left[L_{-1}, L_{-1}\right]=L_{-2}$. In addition, we have that $L_{-1}=\left[L_{-2}, L_{1}\right]$. Lastly, $\left[L_{-1}, x\right] \neq\{0\}$ for all $x \notin L_{-2}$.

Proof. If $\left[x, L_{i}\right]=\{0\}$ for all $i<0$, then

$$
I_{x}=\langle x\rangle+\sum_{\substack{t>0 \\ j_{1}, \ldots, j_{t}>0}}\left[\cdots\left[x, L_{j_{1}}\right], \ldots, L_{j_{t}}\right]
$$

is an ideal of $L$, so that $I_{x}$ equals $L$. Hence, if $x \in L_{j}$ for some $j>-1$, and $\left[x, L_{i}\right]=\{0\}$ for all $i<0$, then we would have $L=I_{x} \subseteq L_{j} \oplus L_{j+1} \oplus \cdots \oplus L_{k}$, a contradiction. It follows that for all $j>-1$, we have that for all nonzero $x \in L_{j}$, the subspace $\left[L_{i}, x\right]$ is nonzero for some $i<0$. In particular, since $\left[L_{-2}, L_{-1}\right]=\{0\}$, we must have $\left[L_{-1}, L_{-1}\right] \neq\{0\}$.

On the other hand, for any $x \in L_{-2}$, we have $I_{x}=L$, so that $L_{-2} \subseteq I_{x}$ (which implies that $L_{-2}$ is irreducible) and $L_{-1} \subseteq I_{x}$ (which implies that $L_{-1}=\left[L_{-2}, L_{1}\right]$ ). Since $\left[L_{-1}, L_{-1}\right]$ is a nonzero submodule of $L_{-2}$, we must have $L_{-2}=\left[L_{-1}, L_{-1}\right]$. Then by the Jacobi identity, we have that $\left[L_{-1}, x\right]=\{0\}$ implies $\left[L_{-2}, x\right]=\{0\}$. Consequently, for any $j>-1$ and any $x \in L_{j}$, we have from the preceding paragraph that $\left[L_{-1}, x\right] \neq\{0\}$. Q.E.D.

LEMMA 2. If $L$ is a simple, graded Lie algebra of the form (1), then $L_{k}$ is an irreducible $L_{0}$-module, and $L_{j-1}=\left[L_{j}, L_{-1}\right]$ for all $j,-1<j<k$. If $L$ is also irreducible, and the characteristic of the base field is greater than 3 , then $L$ is $a$ transitive Lie algebra; that is,

(a) $\left[L_{-1}, w\right] \neq\{0\}$ for all $w \in L_{j^{\prime}} \backslash\{0\}, 0<j^{\prime}<k$, and

(b) $\left[L_{1}, z\right] \neq\{0\}$ for all $z \in L_{j^{\prime \prime}} \backslash\{0\},-2<j^{\prime \prime}<0$.

Proof. If $\left[y, L_{i}\right]=\{0\}$ for all $i>0$, then

$$
J_{y}=\langle y\rangle+\sum_{\substack{t>0 \\ j_{1}, \ldots, j_{t}<0}}\left[\cdots\left[y, L_{j_{1}}\right], \ldots, L_{j_{t}}\right]
$$

is an ideal of $L$, so that $J_{y}$ equals $L$. Since $L_{-2}=\left[L_{-1}, L_{-1}\right]$ by Lemma 1 , we have that

$$
J_{y}=\langle y\rangle+\sum_{\substack{m>0 \\ r>0}}\left[\cdots \left[\left[\cdots[y, \underbrace{\left.\left.L_{0}\right], \ldots, L_{0}\right]}_{m \text { copies }} \underbrace{\left.\left.L_{-1}\right], \ldots, L_{-1}\right]}_{r \text { copies }},\right.\right.\right.
$$

so that $L_{j-1}=\left[L_{j}, L_{-1}\right]$.

It remains to show that $L$ is transitive. Lemma 1 shows that (a) holds. That (b) is true is established by the following proof. 
I. Ann $L_{L^{*}} L_{1}$ is an $L_{0}$-submodule of $L_{j "}$, so by the irreducibility of $L_{-1}$ (by hypothesis) and $L_{-2}$ (by Lemma 1), $A n_{L_{,}} L_{1}=\{0\}$ or $L_{j^{\prime \prime}}$ for $j^{\prime \prime}<0$. By Lemma 1 , $\operatorname{Ann}_{L_{r}} L_{1}=\{0\}$ for $j^{\prime \prime}<0$.

II. Let $B=\operatorname{Ann}_{L_{0}} L_{1}$. If $B \neq\{0\}$, then $\left[L_{-1}, B\right]$ is a nonzero $L_{0}$-submodule of $L_{-1}$, so $\left[L_{-1}, B\right]=L_{-1}$. Then $\left[L_{-1}, L_{1}\right]=\left[\left[L_{-1}, B\right], L_{1}\right] \subseteq\left[\left[L_{-1}, L_{1}\right], B\right]+$ $\left[L_{-1},\left[B, L_{1}\right]\right] \subseteq\left[L_{0}, B\right] \subseteq B$. Then by the first part of this lemma, $B=L_{0}$. Thus, if $y \in L_{1}$, then $\left[L_{-1}, y\right]$ is an ideal of $L_{0}$. But if $x \in L_{-1}$, then $\{0\}=(\operatorname{ad} y)^{3}(\operatorname{ad} x)^{3} L_{0}$ $=6(\operatorname{ad}[y, x])^{3} L_{0}$. Thus, $(\operatorname{ad}[y, x])^{3}=0$. Thus, $\left[L_{-1}, y\right]$ is a nil ideal, and as $L_{0}$ acts faithfully and irreducibly on $L_{-1}$, Engel's Theorem implies that $\left[L_{-1}, y\right]=\{0\}$. Hence, $\left[L_{-1}, L_{1}\right]=\{0\}$, which contradicts (a). Q.E.D.

LEMMA 3. We have $\left[L_{-2}, L_{i}\right] \neq\{0\}$ for all $i, 0<i<k$, and $\left[L_{0}, L_{k}\right] \neq\{0\}$.

Proof. Because for $y \in L_{k}, J_{y}$ above equals $L$, we have from the fact that $\left[L_{-2}, L\right] \neq\{0\}$ that $\left[L_{-2}, L_{k}\right] \neq\{0\}$. Since

$$
\underbrace{L_{-1}, \ldots,\left[L_{-1},\right.}_{k \text { copies }},\left[L_{-2}, L_{k}\right]] \cdots]
$$

is nonzero and equal to

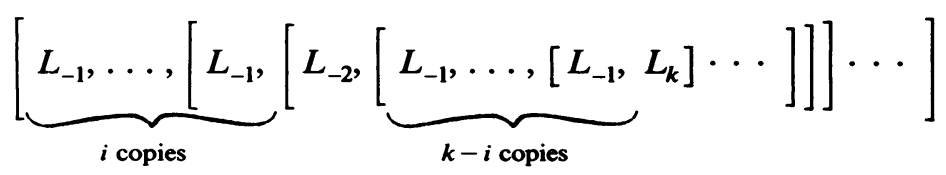

for all $i, 0 \leqslant i \leqslant k$, we have the first assertion of the lemma. The proof that $\left[L_{k}, L_{0}\right] \neq\{0\}$ is similar, using the fact that (by the first part of this lemma) $\left[L_{-2}, L_{k}\right] \neq\{0\}$, together with the fact that for any $y \notin L_{k}$, there exists an $i>0$ such that $\left[L_{i}, y\right] \neq\{0\}$ (see the construction of $J_{y}$ above). Q.E.D.

LEMMA 4. For all $i,-2 \leqslant i \leqslant k-1, L_{i}$ is spanned by weight vectors of the form $[v, x]$, where $v$ is a weight vector for the representation of $L_{0}$ in $L_{-1}$ and $x$ is a weight vector for the representation of $L_{0}$ in $L_{i+1} . L_{k}$ is spanned by weight vectors also.

Proof. In view of Lemma 2 and our assumption that $L_{-1}$ is irreducible as an $L_{0}$-module, this follows as in the proof of Lemma 7 of [1]. Q.E.D.

We denote the annihilator of a submodule $S$ in a submodule $T$ by $\operatorname{Ann}_{T} S$. Then we have the following

Lemma 5. Let $i>0$, and suppose that $\mathrm{Ann}_{L_{i}} L_{-2} \neq\{0\}$. Then $\operatorname{Ann}_{L_{-1}} L_{-2} \neq\{0\}$.

Proof. By Lemma 1, $\left[L_{-1}, \mathrm{Ann}_{L_{i}} L_{-2}\right] \neq\{0\}$. But

$$
\left[L_{-2},\left[L_{-1}, \operatorname{Ann}_{L_{i}} L_{-2}\right]\right]=\left[L_{-1},\left[L_{-2}, \operatorname{Ann}_{L_{i}} L_{-2}\right]\right]=\{0\}
$$

so that $(\{0\} \neq)\left[L_{-1}, \mathrm{Ann}_{L_{i}} L_{-2}\right] \subseteq \mathrm{Ann}_{L_{i-1}} L_{-2}$. Q.E.D.

LEMMA 6. If $\operatorname{Ann}_{L_{1}} L_{-2} \neq\{0\}$, then $L_{-1}$ contains a highest-weight vector $v^{+}$for the representation of $L_{0}$ in $L_{-1}$; i.e., $\left[B^{+}, v^{+}\right] \subseteq\left\langle v^{+}\right\rangle$. 
Proof. Since $A \operatorname{Ann}_{L_{1}} L_{-2}$ is an $L_{0}$-submodule of $L_{1}, M=\left[L_{-1}, \mathrm{Ann}_{L_{1}} L_{-2}\right]$ is an $L_{0}$-submodule of $L_{0} . M$ is nonzero by Lemma 1 , so $M$ is the direct sum of certain of the direct summands of $L_{0}$ (including, possibly, the center). Let $r^{+}$be a root vector of $M$ such that $\left[B^{+}, r^{+}\right] \subseteq\left\langle r^{+}\right\rangle\left(r^{+}\right.$could be a highest-root vector of one of the simple direct summands of $M$, if such there be, or $r^{+}$could be an element of the center of $L_{0}$, if the latter is contained in $M$ ). Then, since the nonzero-root spaces of $L_{0}$ are one-dimensional (and the center of $L_{0}$ is assumed to be at most one-dimensional), we have, in view of the definition of $M$ and Lemma 4, that $r^{+}=[v, a]$, where $v$ and $a$ are weight vectors of $L_{-1}$ and $A \mathrm{An}_{L_{1}} L_{-2}$, respectively. Since $\left[L_{-1}, r^{+}\right] \neq\{0\}$ by Lemma 1 , there exists a weight vector $v_{0} \in L_{-1}$ such that $\left[v_{0}, r^{+}\right] \neq 0$ by Lemma 4 . Because the operators ad $L_{-1}$ commute on $\operatorname{Ann}_{L_{1}} L_{-2}$, the existence of a highest-weight vector for the representation of $L_{0}$ in $L_{-1}$ follows as in the proof of Lemma 8 of [1]. Q.E.D.

LEMMA 7. If $\mathrm{Ann}_{L_{j}} L_{-2}=\{0\}$ for all $j \geqslant 0$, then $L_{-1}$ contains a highest-weight vector $v^{+}$for the representation of $L_{0}$ in $L_{-1}$.

Proof. Since $\mathrm{Ann}_{L_{0}} L_{-2}=\{0\}$, an argument similar to that used in the proof of Lemma 8 of [1] shows that $L_{-2}$ contains a highest-weight vector for the representation of $L_{0}$ in $L_{-2}$ (begin the argument by choosing $r^{+} \in\left[L_{-2}, L_{2}\right]$, which is nonzero by Lemma 3). Then an argument similar to that used in $\$ 4$ of [1] shows that the representation of $L_{0}$ in $L_{-2}$ is restricted. Thus, in view of Lemma 1 and Kac's remark in $\$ 5$ of [2],

$$
E=L_{-2} \oplus L_{0} \oplus L_{2} \oplus \cdots \oplus L_{2[k / 2]}
$$

is an irreducible, transitive, graded Lie algebra such that the representation of $L_{0}$ in $L_{-2}$ is restricted. Then Theorem 3 of [2] shows that $E$ is classical or of Cartan type.

If $k>3$, then $2[k / 2] \geqslant 4$, so $E$ must be of Cartan type, and $L_{0}$ is the direct sum of its (possibly trivial) center and a simple Lie algebra $S$ of type $A_{n}$ or $C_{n}$; furthermore, the representation of $S$ on $L_{-2}$ is the natural representation of $S$.

Using Lemma 2, we can write, as before, $r^{+}=[v, c]$, where $r^{+}$is a highest-root vector of $S$, and where $v$ and $c$ are weight vectors for the representations of $L_{0}$ in $L_{-1}$ and $L_{1}$, respectively. Since $\left[L_{-1}, r^{+}\right] \neq\{0\}$, we know that there exists a weight vector $v_{0}$ in $L_{-1}$ such that $\left[v_{0}, r^{+}\right] \neq 0$. Suppose there were an infinite sequence of vectors $e_{i_{1}}, e_{i_{2}}, \ldots$, associated with the simple (positive) roots $\alpha_{i_{1}}, \alpha_{i_{2}}, \ldots$, respectively, such that

$$
v_{j}^{\prime}=\left[\cdots\left[\left[\left[v_{0}, r^{+}\right], e_{i_{1}}\right], e_{i_{2}}\right], \ldots, e_{i_{j}}\right] \neq 0
$$

for all $j \geqslant 1$. If we define $v_{j}=\left[\cdots\left[v_{0}, e_{i_{1}}\right], \ldots, e_{i_{j}}\right]$, we have $(0 \neq) v_{j}^{\prime}=\left[v_{j}, r^{+}\right]$ $=\left[\left[v_{j}, v\right], c\right]+\left[v,\left[v_{j}, c\right]\right]$ for all $j \geqslant 1$; i.e., for all $j \geqslant 1$, either $\left[v_{j}, v\right] \neq 0$ or $\left[v_{j}, c\right] \neq 0$. Let $w_{0}$ be the sum of the weights of $v_{0}$ and $v$, and let $\beta_{0}$ be the sum of the weights of $v_{0}$ and $c$. For $j \geqslant 1$, we recursively define $w_{j}=w_{j-1}+\alpha_{i j}$ and $\beta_{j}=\beta_{j-1}+\alpha_{i j}$. Then when $\left[v_{j}, v\right]$ is nonzero, it has weight $w_{j}$; similarly, when $\left[v_{j}, c\right]$ is nonzero, it has $\operatorname{root} \beta_{j}$. 
Now suppose that the weight vectors $\left\{\left[v_{j+i}, v\right]\right\}_{i=0}^{t}$ are all nonzero, and let $w_{j}, w_{j+1}, \ldots, w_{j+\ell}$ be their corresponding weights. Let $w_{A}^{-}, w_{A}^{+}, w_{C}^{-}$, and $w_{C}^{+}$be the lowest and highest weights of $A_{n}$ and $C_{n}$, respectively, and set

$$
\begin{aligned}
w_{1}^{(1)} & =w_{A}^{-}, \quad w_{2}^{(1)}=w_{A}^{-}+\alpha_{n}, \\
w_{3}^{(1)} & =w_{A}^{-}+\alpha_{n}+\alpha_{n-1}, \ldots, \\
w_{n+1}^{(1)} & =w_{A}^{-}+\alpha_{n}+\alpha_{n-1}+\cdots+\alpha_{1}=w_{A}^{+} ; \\
w_{1}^{(2)} & =w_{A}^{-}, \quad w_{2}^{(2)}=w_{A}^{-}+\alpha_{1}, \\
w_{3}^{(2)} & =w_{A}^{-}+\alpha_{1}+\alpha_{2}, \ldots, \\
w_{n+1}^{(2)} & =w_{A}^{-}+\alpha_{1}+\alpha_{2}+\cdots+\alpha_{n}=w_{A}^{+} ; \\
w_{1}^{(3)} & =w_{C}^{-}, \quad w_{2}^{(3)}=w_{C}^{-}+\alpha_{1}, \\
w_{3}^{(3)} & =w_{C}^{-}+\alpha_{1}+\alpha_{2}, \cdots, \\
w_{2 n}^{(3)} & =w_{C}^{-}+\alpha_{1}+\alpha_{2}+\cdots+\alpha_{n-1}+\alpha_{n}+\alpha_{n-1}+\cdots+\alpha_{1}=w_{C}^{+} .
\end{aligned}
$$

Since $w_{b}^{(d)} \neq w_{X}^{+}+\alpha_{l}, X=A$ or $C$, for all integers $b, d$, and $l$ for which the expression is defined, it follows that if $S=A_{n}$, we must have an integer $m$ such that $w_{j+i}=w_{m+i}^{(1)}, i=0, \ldots, t$, or $w_{j+i}=w_{m+i}^{(2)}, i=0, \ldots, t$; similarly, if $S=C_{n}$, we must have an $m$ such that $w_{j+i}=w_{m+i}^{(3)}, i=0, \ldots, t$. In particular, it must eventually occur that, for some integer $s, w_{s}$ is a weight, $w_{s+1}$ is not a weight, and if $q$ is the smallest positive integer such that $w_{s+q}$ is a weight, then $w_{s}=w_{b}^{(d)}$ and $w_{s+q}=w_{b^{\prime}}^{(d)}$, where $b^{\prime} \leqslant b\left(d=1,2\right.$, or 3). Then $\beta_{s+1}, \beta_{s+2}, \ldots, \beta_{s+q-1}$ must be a root string of $L_{0}$ such that $w_{s+q}-w_{s}=\alpha_{i_{s+1}}+\left(\beta_{s+q-1}-\beta_{s+1}\right)+\alpha_{i_{s+q}}$. In view of (2), (3), and (4), $-\alpha_{i}$ can appear no more than twice as a summand of $w_{s+q}-w_{s}$ for any $i, 1 \leqslant i \leqslant n$. Thus, $\alpha_{i_{s+1}}+\left(\beta_{s+q-1}-\beta_{s+1}\right)+\alpha_{i_{s+q}}=\sum_{i=1}^{n} t_{i} \alpha_{i}$, where $t_{i} \equiv p-$ $2, t_{i} \equiv p-1$, or $t_{i} \equiv 0(\bmod p)$. Then $\beta_{s+q-1}-\beta_{s+1}$ must contain at least $p-4>$ 3 copies of each simple root appearing in it. Inasmuch as $\beta_{s+q-1}-\beta_{s+1}$ is the sum of the roots in at most two contiguous subarrays of the following array:

$$
\begin{aligned}
& \alpha_{1} \alpha_{2} \cdots \alpha_{n-1} \\
& \alpha_{1} \alpha_{2} \cdots \alpha_{n-1}
\end{aligned}
$$

it is clear that we have arrived at a contradiction. (Note that if any contiguous subarray contains more than two copies of the same simple root, then it must be a subarray of the second array and contain at most two copies of $\alpha_{n}$.) Thus, no such infinite sequence as $\left\{\left[v_{j}, r^{+}\right]\right\}_{j=1}^{\infty}$ can occur, and we must arrive at a vector $v^{+}=\left[v_{u}, r^{+}\right], u \geqslant 1$, which is annihilated by $\left\{e_{i}\right\}_{i=1}^{n}$.

Now suppose that $k=3$. By the above-quoted Theorem 3 of [2], there exists an element $d^{+}$of $L_{2}$ which is a highest-root vector of the classical Lie algebra $E$, and, hence, a highest-weight vector for the representation of $L_{0}$ in $L_{2}$. By Lemma 2 (and the fact that the nonzero-root spaces of a classical Lie algebra are onedimensional), it follows that $d^{+}=[v, e]$, where $v$ and $e$ are weight vectors of $L_{-1}$ and $L_{3}$, respectively. Letting $y=d^{+}$in the proof of Lemma 2, we get that $\left[d^{+}, L_{1}\right] \neq\{0\}$. Let $c$ be a weight vector of $L_{1}$ such that $e^{\prime}=\left[d^{+}, c\right] \neq 0$, and 
suppose there existed an infinite sequence of simple (positive) root vectors $e_{i}$, $e_{i_{2}}, \ldots$, such that $\left[\cdots\left[e^{\prime}, e_{i_{1}}\right], \ldots, e_{i_{j}}\right] \neq 0$ for all $j>1$. Then

$$
\begin{aligned}
(0 \neq)\left[\cdots\left[e^{\prime}, e_{i_{1}}\right], \ldots, e_{i_{j}}\right] & =\left[\cdots\left[\left[d^{+}, c\right], e_{i_{1}}\right], \ldots, e_{i_{j}}\right] \\
& =\left[d^{+},\left[\cdots\left[c, e_{i_{1}}\right], \ldots, e_{i_{j}}\right]\right] \\
& =\left[[v, e],\left[\cdots\left[c, e_{i_{1}}\right], \ldots, e_{i_{j}}\right]\right] \\
& =\left[\left[v,\left[\cdots\left[c, e_{i_{1}}\right], \ldots, e_{i j}\right]\right], e\right]
\end{aligned}
$$

for all $j \geqslant 1$. But then $\left\{\left[v,\left[\cdots\left[c, e_{i_{1}}\right], \ldots, e_{i_{i}}\right]\right]\right\}_{j=1}^{\infty}$ in an infinite sequence of root vectors of $L_{0}$, each of whose roots differs from the previous root by a simple positive root. Since such a sequence cannot exist in the classical $L_{0}$, we see that $L_{3}$ must contain a highest-weight vector $e^{+}$. As we noted at the beginning of the proof of this lemma, $L_{-2}$ contains a highest-weight vector $w^{+}$. Since we have assumed that $\mathrm{Ann}_{L_{3}} L_{-2}=\{0\}$, we have that $\left[L_{-2}, e^{+}\right] \neq\{0\}$. By Lemma $1, L_{-2}$ is irreducible as an $L_{0}$-module, so it is spanned by vectors of the form $\left[\cdots\left[w^{+}, f_{i_{1}}\right] \ldots, f_{i j}\right]$. Let $j$ be minimal such that $c^{+}=\left[\left[\cdots\left[w^{+}, f_{i_{1}}\right], \ldots, f_{i_{j}}\right], e^{+}\right] \neq 0$. Then $c^{+}$is a highest-weight vector of $L_{1}$. Similarly, if $j^{\prime}$ is minimal such that $v^{+}=$ $\left[\left[\cdots\left[w^{+} f_{i_{1}^{\prime}}\right], \ldots, f_{i^{\prime}}\right], c^{+}\right] \neq 0$, then $v^{+}$is a highest-weight vector of $L_{-1}$, as required. Q.E.D.

LEMMA 8. If $\mathrm{Ann}_{L_{i}} L_{-2}=\{0\}$ for all $i>1$, and $\mathrm{Ann}_{L_{0}} L_{-2} \neq\{0\}$, then $L_{-1}$ contains a highest-weight vector $v^{+}$for the representation of $L_{0}$ in $L_{-1}$.

Proof. Set $A=\operatorname{Ann}_{L_{0}} L_{-2}$. Since $\operatorname{Ann}_{L_{i}} L_{-2}=\{0\}$ for all $i>1$, we have that [ $\left.\left[L_{3}, L_{-2}\right], L_{-2}\right] \neq\{0\}$. Since $L_{-1}$ is assumed to be irreducible, we have that $L_{-1}=$ [[ $\left.\left.L_{3}, L_{-2}\right], L_{-2}\right]$. By Lemma 2, we have that $L_{0}=\left[L_{-1}, L_{1}\right]$, so

$$
\begin{aligned}
L_{0} & =\left[L_{-1}, L_{1}\right]=\left[\left[\left[L_{3}, L_{-2}\right], L_{-2}\right], L_{1}\right] \\
& \subseteq\left[\left[\left[L_{3} L_{1}\right], L_{-2}\right], L_{-2}\right]+\left[\left[L_{3},\left[L_{-2}, L_{1}\right]\right], L_{-2}\right]+\left[\left[L_{3}, L_{-2}\right],\left[L_{-2}, L_{1}\right]\right] \\
& \subseteq\left[\left[L_{4}, L_{-2}\right], L_{-2}\right]+\left[\left[L_{3}, L_{-1}\right], L_{-2}\right]+\left[\left[L_{3}, L_{-2}\right], L_{-1}\right] \\
& =\left[\left[L_{4}, L_{-2}\right], L_{-2}\right]+\left[\left[L_{3}, L_{-1}\right], L_{-2}\right]+\left[\left[L_{3}, L_{-1}\right], L_{-2}\right] \\
& \subseteq\left[L_{2}, L_{-2}\right] .
\end{aligned}
$$

Hence, $L_{0}=\left[L_{2}, L_{-2}\right]$.

Now consider $D=\left[A, L_{2}\right]$. We have

$$
\left[L_{-2}, D\right]=\left[L_{-2},\left[A, L_{2}\right]\right]=\left[A,\left[L_{-2}, L_{2}\right]\right]=\left[A, L_{0}\right]=A \neq\{0\}
$$

( $A$ cannot contain the center, since each nonzero element of the center acts as a nonzero scalar on $L_{-2}$ ). Let $r^{+}$be a highest-root vector of $A$. Then, as before, $r^{+}=[w, d]$, where $w$ is a weight vector of $L_{-2}$ and $d$ is a weight vector of $D$. Furthermore, since every element of $L_{-2}$ has $A$-weight zero, $r^{+}$and $d$ have the same $A$-weight. Since $L_{-2}=\left[L_{-1}, L_{-1}\right]$ by Lemma 1 , it follows that $w$ is a linear combination of weight vectors of the form $\left[v, v^{\prime}\right]$, where $v$ and $v^{\prime}$ are weight vectors of $L_{-1}$. Since the nonzero-root spaces of $L_{0}$ are one-dimensional, we must have that 
$r^{+}=\left[\left[v, v^{\prime}\right], d\right]$ for certain $v$ and $v^{\prime}$. By the Jacobi identity, $r^{+}=\left[v,\left[v^{\prime}, d\right]\right]-$ $\left[v^{\prime},[v, d]\right]$, so that by relabelling and/or multiplying one of the vectors $v$ or $v^{\prime}$ by a scalar, we can assume that $r^{+}=\left[v,\left[v^{\prime}, d\right]\right]$. Since $r^{+}$and $d$ have the same $A$-weight, the $A$-weight of $v$ must be the negative of the $A$-weight of $v^{\prime}$. Now, by Lemma $1,\left[L_{-1}, r^{+}\right] \neq\{0\}$, so there exists a $v_{0} \in L_{-1}$ such that $\left[v_{0}, r^{+}\right]=$ $\left[v_{0},\left[v,\left[v^{\prime}, d\right]\right]\right] \neq 0$. Suppose there were an infinite sequence $e_{i_{1}}, e_{i_{2}}, \ldots$ of simple (positive) root vectors such that

$$
v_{j}^{\prime}=\left[\cdots\left[\left[v_{0}, r^{+}\right], e_{i_{1}}\right], \ldots, e_{i_{j}}\right] \neq 0 \text { for all } j>1 .
$$

If we define $v_{j}=\left[\cdots\left[v_{0}, e_{i_{1}}\right], \ldots, e_{i j}\right]$, then we have

$$
(0 \neq) \quad v_{j}^{\prime}=\left[v_{j}, r^{+}\right]=\left[\left[v_{j}, v\right],\left[v^{\prime}, d\right]\right]+\left[v,\left[v_{j},\left[v^{\prime}, d\right]\right]\right] \text {. }
$$

Set $s_{j}=\left[\left[v_{j}, v\right],\left[v^{\prime}, d\right]\right]$ and $t_{j}=\left[v,\left[v_{j},\left[v^{\prime}, d\right]\right]\right]$. Then for all $j>1$, either $s_{j} \neq 0$ or $t_{j} \neq 0$.

If $s_{j} \neq 0$, then $\left[v_{j}, v\right] \neq 0$. But $\left[v_{j}, v\right] \in L_{-2}$, so $\left[v_{j}, v\right]$ has zero $A$-weight. Thus, the $A$-weight of $v_{j}$ must be the negative of that of $v$; i.e., the $A$-weight of $v_{j}$ must equal the $A$-weight of $v^{\prime}$. Now, $(0 \neq) s_{j}=\left[\left[v_{j}, v\right],\left[v^{\prime}, d\right]\right]=\left[v^{\prime},\left[\left[v_{j}, v\right], d\right]\right]$. Furthermore, by (5), $\left[\left[v_{j}, v\right], d\right] \in A$, and since $\left[\left[v_{j}, v\right], d\right]$ has the same $A$-weight as $\left[v,\left[v^{\prime}, d\right]\right]=r^{+}$, we must have $(0 \neq)\left[\left[v_{j}, v\right], d\right] \in\left\langle r^{+}\right\rangle$.

Thus, $v_{j}$ has the same $L_{0}$-weight as $v^{\prime}$; that is, the only occasion on which $s_{j} \neq 0$ is when $v_{j}$ has the same $L_{0}$-weight as $v^{\prime}$.

If $t_{j} \neq 0$, then $r_{j}=\left[v_{j},\left[v^{\prime}, d\right]\right]$ is a nonzero-root vector of $L_{0}$. But by the preceding two paragraphs, $t_{j}=0$ only when $v_{j}$ has $L_{0}$-weight equal to $v^{\prime}$. Thus, if $j^{\prime}$ is the first subscript for which $t_{j^{\prime}}=0$ and $j^{\prime \prime}$ is the second, then the $L_{0}$-weight of $v_{j^{\prime}}$ is equal to the $L_{0}$-weight of $v_{j^{\prime \prime}}$, so that we have a sequence of root vectors $r_{j^{\prime}+1}, r_{j^{\prime}+2}, \ldots, r_{j^{\prime \prime}-1}$ of $L_{0}$ such that the root associated with $r_{j^{\prime}+1}$ differs (modulo $p$ ) from the root associated with $r_{j^{\prime \prime}-1}$ by the sum of two simple roots $\left(\alpha_{j^{\prime}+1}\right.$ and $\left.\alpha_{j^{\prime \prime}}\right)$. Thus, each simple (positive) root in the sequence $\alpha_{j^{\prime}+2}, \ldots, \alpha_{j^{\prime \prime}-1}$ appears at least $p-2 \geqslant 5$ times. Since no such root string can occur in a classical Lie algebra, no such sequence as $\left\{v_{j}^{\prime}\right\}_{i=1}^{\infty}$ can occur; i.e., any such sequence must terminate in a vector $v^{+}$which is annihilated by all of $\left\{e_{i}\right\}_{i=1}^{n}$. Then $v^{+}$is the required highestweight vector. Q.E.D.

Proof of TheOREM. By Lemmas 5, 6, 7, and 8, we have that $L_{-1}$ contains a highest-weight vector for the representation of $L_{0}$ in $L_{-1}$. Then the theorem follows as in $\$ 4$ of [1]. Q.E.D.

The author wishes to express his gratitude to Professor Joseph C. Ferrar for his generous interest in the author's work and for his encouraging suggestions.

\section{REFERENCES}

1. T. B. Gregory, Simple Lie algebras with classical reductive null component, J. Algebra 63 (1980), 484-493.

2. V. G. Kac, The classification of the simple Lie algebras over a field with non-zero characteristic, Izv. Akad. Nauk SSSR Ser. Mat. 34 (1970), 385-408; .English transl., Math USSR Izv. 4 (1970), 391-413.

Department of Mathematics, The Ohio State University at Mansfield, Mansfield, Ohio 44906 